\title{
A Simple Fusion Method for Trajectory Data of Aerocraft
}

\author{
Dingxin Yang*, Peng Du and Jinwei Sun \\ China Satellite Measure and Track Centre, China Jiangyin \\ ${ }^{*}$ Corresponding author
}

\begin{abstract}
Propose a fusion arithmetic for trajectory data of aerocraft in flight. The method uses a variety of trajectory data which we can get for fusion calculation, then we can reduce a compositive trajectory data for digital-guided, and as the basis of judging the aerocraft flight state. Through the past, an active phase of the aerocraft tracking data playback, testified the practicability and effectiveness.
\end{abstract}

\section{Keywords-trajectory; fusion; support}

\section{INTRODUCTION}

In the process of aerocraft in flight, the measured data of aerocraft trajectory is adopted for the digital guiding of measuring devices, and it is also a major basis for estimating the trajectory of aerocrafts. With the ever increasing developments of astronautics measuring techniques, there are a variety of external trajectory procedures which make various trajectory measuring sources available. Thus we have more choices of trajectory data utilization. Aerocraft launching tasks are short in time and demands real-time property of data measuring. It requires stable and precise delivery of digital guiding data, together with reliable estimation of trajectory data Existing methods are mainly based on human jobs including comparing the target trajectory and the theoretical trajectory, and observing the curves of trajectory speed and height is also necessary. Since human jobs are involved, this process takes long time and becomes unreliable. Once errors occur, the digital guiding data will be quite unstable and the secure of measuring devices are influenced. This paper proposes a integration method for trajectory data, and it computes a systematic trajectory dataset based on integration of the retrieved trajectory data. Our method improves the reliability of trajectory significantly.

\section{DATA INTEGRATION ALGORITHM}

After measuring devices obtain $m$ measuring values denoted $x_{i}(i=1,2, \ldots \mathrm{n})$, the integration method utilizes these values in a synthetical manner [1]. Here $x_{i}$ does not follow the normal distribution due to the computation error, noises, measuring conditions, precision of measuring devices and certain factors of the measured objects. So data processing methods based on the normal distribution model will suffer from systematic error [2]. In reality, the quality of measured data is decided by facticity of $x_{1}, x_{2}, \ldots, x_{n}$. The better the facticity of $x_{i}$ is, the more support it gets from the other data. The support provided by $x_{j}$ to $x_{i}$ can be considered as the confidence of $x_{i}$ judged by $x_{j}$. Here we denote the support between different data items as a relative distance. The definition of the relative distance $d_{i j}$ is given as in (1):

$$
d_{i j}=\left|x_{i}-x_{j}\right|
$$

As shown in equation (1), the larger $d_{i j}$ is, the more differences exist between $x_{j}$ and $x_{i}$, and the less support we get. The relative distance adopt available measured data, and requires no priori information. Then we define a supporting function $r_{i j}$ :

$$
r_{i j}=-\frac{d_{i j}}{\max \left\{d_{i j}\right\}}+1
$$

Here $\max \left\{\mathrm{d}_{i j}\right\}$ is the maximal value among all the relative distances, and the larger a relative distance is, the less the support provided. As depicted in equation (2), the support value is zero when the maximal relative distance is reached, and here we say there is no support between data items. In opposite, a data item provides a support value as 1 to itself, since the relative distance is valued 0 .

This function provides two properties:

1) The function value is opposite to the relative distances;

2) Since the function value is in $[0,1]$, the advantages of membership function in the fuzzy set theory can be used in the data processing phase, and absolution of support between different data items can be avoided. This improves the precision and reliability of data integration result.

Given the problem of data integration, we conduct a supporting matrix R as in (3):

$$
R=\left[\begin{array}{llll}
r_{11} & r_{12} & \cdots & r_{1 n} \\
r_{21} & r_{22} & \cdots & r_{2 n} \\
\vdots & \vdots & \vdots & \vdots \\
r_{n 1} & r_{n 2} & \cdots & r_{n n}
\end{array}\right]
$$

The value $r_{i j}$ is the supporting value of $x_{i}$ and $x_{j}$, so $R$ is a symmetric matrix, and it can not express the total support from 
a measured data item. Thus we define weight coefficient $\bar{\omega}_{i}$ $\left(\bar{\omega}_{i} \geq 0\right)$, where $\sum_{i=1}^{n} \bar{\omega}_{i}=1$. Take $\bar{\omega}_{i}, r_{i 1}, r_{i 2}, \ldots r_{\text {in }}$ into consideration together, then we define a group of none negative parameters $v_{1}, v_{2}, \ldots v_{n}$ so that

$$
\bar{\omega}_{i}=v_{1} r_{i 1}+v_{2} r_{i 2}+\cdots+v_{n} r_{i n}
$$

In the form of matrix, we have

$$
W=R V
$$

$$
\begin{aligned}
& \text { Here we } W=\left[\bar{\omega}_{1}, \bar{\omega}_{2}, \cdots, \bar{\omega}_{n}\right]^{T} \\
& \text { and } V=\left[v_{1}, v_{2}, \cdots, v_{n}\right]^{T} \text {. Since } r_{i j \geq 0} \text {, the matrix } R \text { is a none } \\
& \text { negative matrix so it has a maximal eigenvalue } \lambda \geq 0 \text {, } \\
& \text { and } \lambda V=R V \text {. Then we can get the corresponding feature } \\
& \text { vector } V=\left[v_{1}, v_{2}, \cdots, v_{n}\right]^{T} \text {. Suppose that }
\end{aligned}
$$

$$
\bar{\omega}_{i}=\frac{v_{i}}{v_{1}+v_{2}+\cdots+v_{n}}
$$

Then $\bar{\omega}_{i}$ is the ith weight coefficient of the measured data item $\mathrm{x}_{\mathrm{i}}$, and integration result of the $n$ measured data items is given as in (7):

$$
x=\bar{\omega}_{1} x_{1}+\bar{\omega}_{2} x_{2}+\cdots+\bar{\omega}_{n} x_{n}
$$

This method is suitable to integrate more than three types of measured data, and it provides advantages as below:

1) Real-time property and feasible computation;

2) Only measured data is required and no priori information is necessary, where existing methods demand priori information of measuring devices [5][6];

3) The more types of measured data, the better quality of integration results.

\section{IMPLEMENTATION AND APPLICATION}

\section{A. Implementation}

The trajectory of aerocraft consists of seven elements as denoted by $r=\{t, x, y, z, v x, v y, v z\}$. Here $t$ is the time, and $\mathrm{x}, \mathrm{y}$, $\mathrm{z}$ are the axis values in 3-d space [3]. At the same time, $v x, v y$, $v Z$ are the speed values along 3-d axis directions. We get a group of trajectory data with the measured trajectory data integration algorithm introduced in the last section. And the result is used for digital guiding, monitor display and state judgement.
The detailed workflow is shown in figure 1.

1) Receive different types of trajectory data in a real-time manner;

2) Align all the measured trajectory data in terms of time and space, and interpolation algorithms are adopted. In this process, all the trajectory data are transformed into a single coordinate system.

3) If we have more than three types of trajectory data, utilize data integration algorithm to obtain a group of systematic trajectory output. More details are referred to [4].

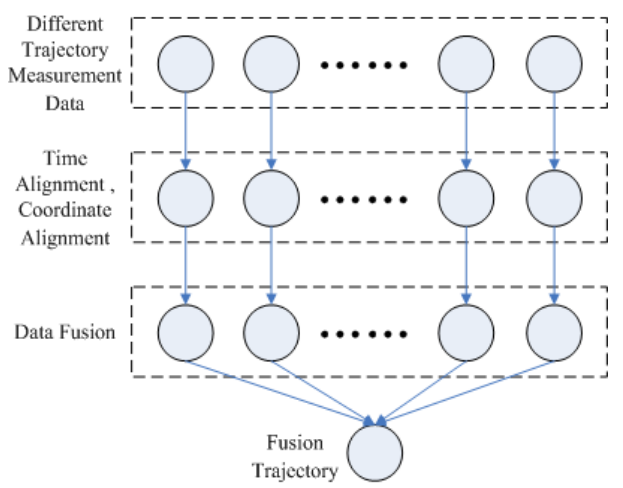

FIGURE I.

THE FRAMEWORK OF IMPLEMENTATION

\section{B. Application}

We implement our method using $\mathrm{VC}++6.0$, and a project for integration computation of aerocraft launch trajectory data is conducted. The algorithm details are referred to [4]. We simulate the aerocraft launch task with historical record data, and get four types of measured trajectory data denoted by A, B, $\mathrm{C}$ and $\mathrm{D}$. We only list six axis values of a trajectory as $(\mathrm{x}, \mathrm{y}, \mathrm{z}$, $\mathrm{v}_{x}, \mathrm{v}_{y}, \mathrm{v}_{z}$ ) due to the limit of space, and Some of the result is shown in figure 2 .

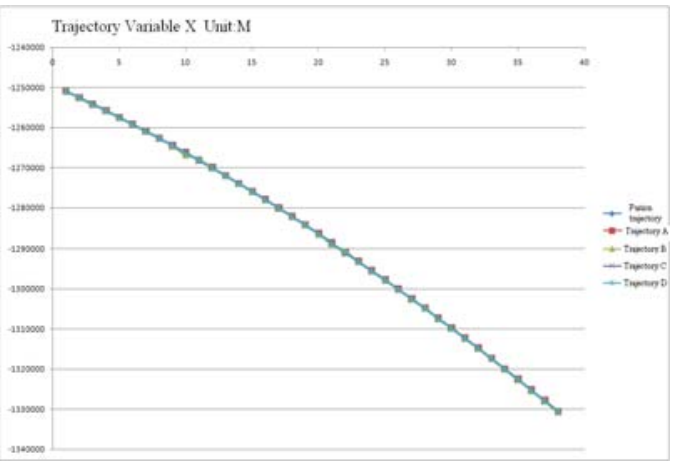




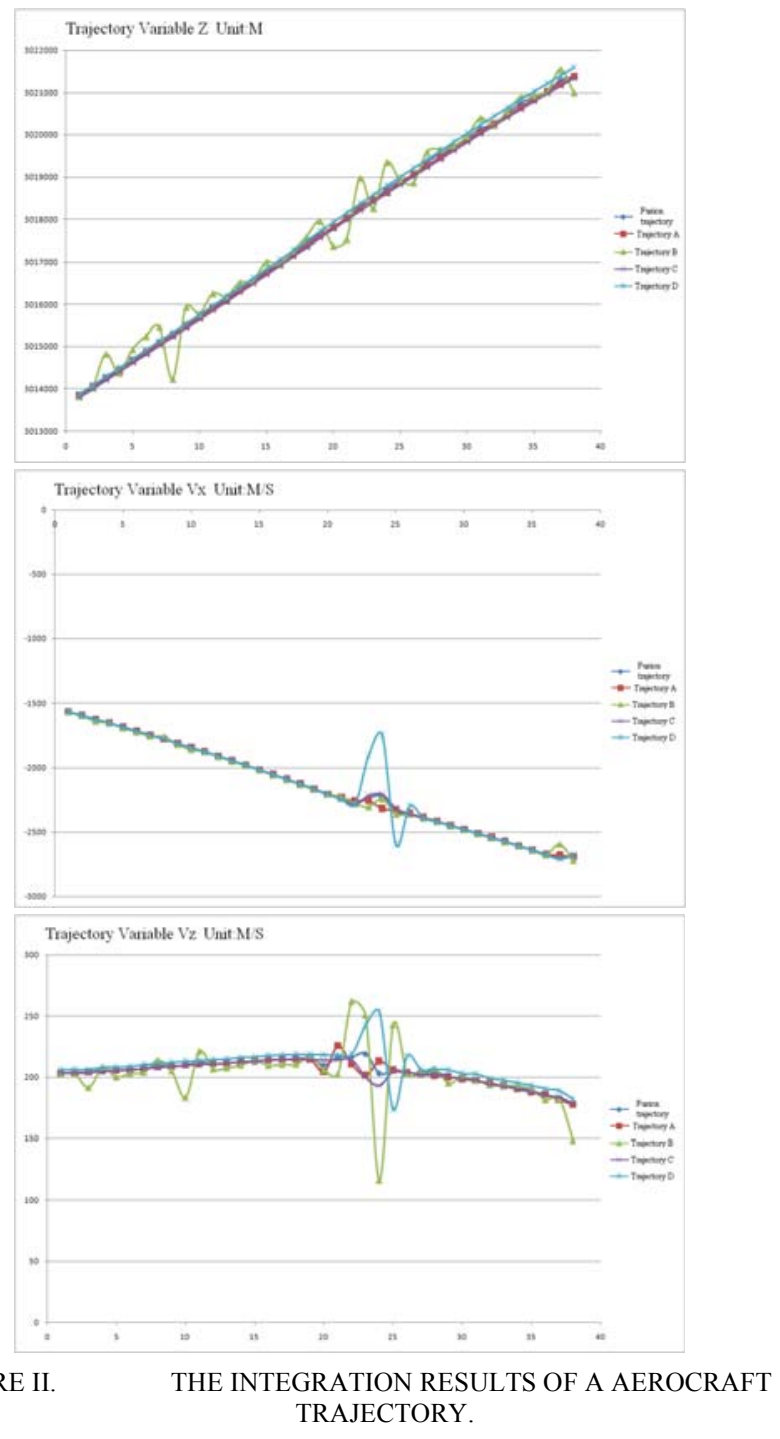

As shown in the measured results, the algorithms are able to integrate all the measured data, and the variables are suitable. This method provides precision, and it gives smooth result when certain types of data are shaking. So we conclude that our method has reasonable effectiveness and efficiency, and it improves the reliability of trajectory data utilization.

\section{CONCLUSION}

We repeat with task record data, and it is shown that our method is able to integrate trajectory data while providing realtime property. This method still needs further verification in more complex environments, since the aerocraft launching process is complex and of unknown factors. What is more, our method can be used in other applications which are in demand of data integration.

\section{REFERENCES}

[1] Dai YP. Multi sensor data fusion theory and Application[M]. Beijing Institute of Technology press, Beijing, China. 2004: 37-61.
[2] Sha DG. Evaluation of uncertainty in measurement and analysis of error[M]. China metrology press, Beijing, China. 2003: 47-66.

[3] $\mathrm{Hu}$ SL. Pulse radar tracking measurement data processing technology[M]. National Defence Industry Press, Beijing, China. 2007:20-34.

[4] Xu SL. Common algorithms for assembly (C++ language description) (Fourth Edition)[M]. Tsinghua University press, Beijing, China. 2008: 95-114.

[5] Xiang SL. Application of the technology of tracking real-time fusion[J]. Chinese radar. 2008(4): 1-3.

[6] Wang Zhaogang, Wu Yinlin. Data Fusion to Ballistic Trajectory Estimation Based on The Least Square Estimate for Multi photo electric Cinetheodolite Intersection [J]. Journal of Projectiles, Rockets, Missiles and Guidance. 2011:31(3): 172-176. 